\title{
Frequency Response of Intracavity Laser Coupling Modulation
}

\author{
AMNON YARIV, THOMAS A. NUSSMEIER, AND JAMES E. KIEFER
}

\begin{abstract}
A resonant energy coupling between the atomic system and the oscillating optical mode leads to severe output distortion in intracavity laser coupling modulation. This anomalous behavior, which places a lower limit on the modulation frequency, is investigated in a case of a $\mathrm{CO}_{2}$ laser and compared with theoretical predictions.
\end{abstract}

\section{INTRODUCTION}

$\mathbf{T}$ HE advantages of intracavity coupling modulation as a means of efficient broad-band laser modulation are by now well established [1]-[5]. These are due primarily to the fact that the modulator operates on the internal circulating power of the laser and thus requires significantly less driving power than for similar performance with the modulator located outside the laser cavity. Furthermore, there are no fundamental limitations on high-frequency performance other than those imposed by the modulator structure itself. At low frequencies, however, an internal modulator will invariably interfere with the interaction process between the atomic system and the optical resonant mode. This may lead under certain conditions to strong fluctuations in the internal power and hence to modulation distortion. A proper design of internally modulated lasers requires an understanding of this phenomenon and its parametric dependences.

In this paper, we present an analysis of this effect as well as the results of a series of experiments that are used to check the theory and illustrate the practical problems encountered in this type of modulation.

\section{THEORY}

The main features of the internal coupling modulator are shown in Fig. 1. An electrooptic crystal placed inside the laser resonator transfers in each round trip a fraction equal to $\sin ^{2} \Gamma$ of the internal circulating power from the dominant polarization (in the plane of the figure) to the orthogonal polarization state. $\Gamma$ is the single-pass electrooptic retardation that can be written as

$$
\Gamma(t)=\frac{V_{0}}{V_{\pi}} \pi+\frac{V_{1}}{V_{\pi}} \pi \cos \omega t \equiv \Gamma_{0}+\Gamma_{1} \cos \omega t,
$$

where $V_{\pi}$ is the "half-wave" voltage of the electrooptic crystal, $V_{0}$ is a dc bias voltage, and $V_{1} \cos \omega t$ is the signal

\footnotetext{
Manuscript received January 16, 1973; revised February 15, 1973.

A. Yariv is with the Department of Electrical Engineering, California Institute of Technology, Pasadena, Calif. 91109.

T. A. Nussmeier and J. E. Kiefer are with the Hughes Research Laboratories, Malibu, Calif. 90265.
}

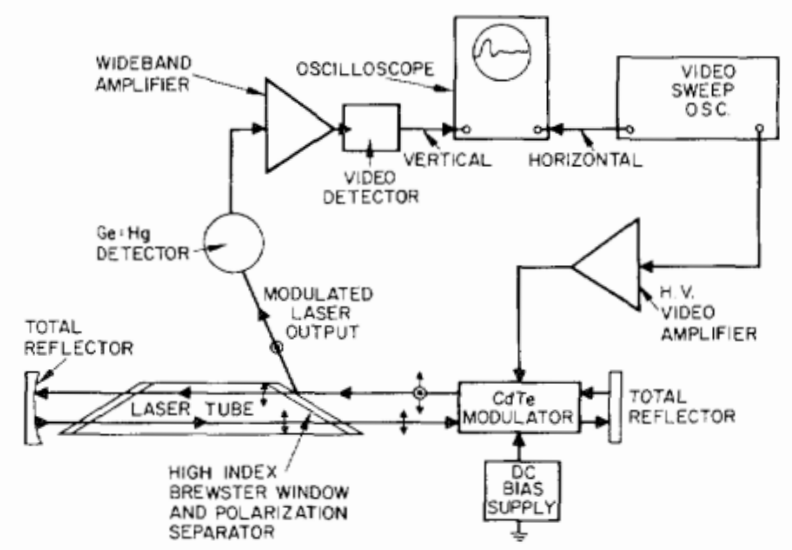

Fig. 1. Experimental arrangement for coupling modulation measurements.

voltage. The orthogonally polarized wave is assumed to be coupled completely out of the optical resonator by means of a polarizer such as, for example, the high-index Brewster-angle window shown in Fig. 1. The coupled beam constitutes the useful power output of the laser. The form of (1) makes no allowance for the frequency dependence of the modulation element itself since $\Gamma_{1}$ is taken to be proportional to the modulation voltage $V_{1}$. This places an upper limit on the modulation frequency, which for most cases will be determined by the transit time through the crystal.

The combination of an electrooptic crystal and internal polarization coupler is thus equivalent to a mirror with an instantaneous transmission

$$
T(t)=\sin ^{2} \Gamma \approx \Gamma_{0}{ }^{2}+2 \Gamma_{0} \Gamma_{1} \cos \omega t+\Gamma_{1}{ }^{2} \cos ^{2} \omega t,
$$

where we allowed for a double traverse of the crystal and assumed $\Gamma(t)<<1$, a condition that is obeyed in practical situations in order to avoid excessive loading of the laser.

An alternative point of view and one that will be used in the following is to represent the modulator-polarizer combination by an effective photon decay rate

$\frac{1}{t_{c}(t)}=\frac{c T(t)}{L} \cong \frac{c \Gamma_{0}{ }^{2}}{L}+\frac{2 c \Gamma_{0} \Gamma_{1}}{L} \cos \omega t+\frac{c \Gamma_{1}{ }^{2}}{L} \cos (2 \omega t)$,

where $c$ is the velocity of light and $L$ is the length of the resonator. In the following, we limit the discussion to the case $\Gamma_{0} \gg \Gamma_{1}$ and neglect the last term in (3). This limits the analysis to the small-signal regime case where the modulated portion of the output power is small compared 
to its average value. This limitation, however, is not expected to modify substantially the results reached below. The inclusion of this term when $\Gamma_{0}$ and $\Gamma_{1}$ are comparable, does not change significantly any of the results reached below.

Our first task is to solve for the total stored energy in the optical resonator in the presence of the time-varying losses represented by (3). We start with the rate equations for the total number of photons in the resonator $q$ and the (total) atomic inversion $N$

$$
\begin{aligned}
& \frac{d N}{d t}=R-B q N-\frac{N}{\tau} \\
& \frac{d q}{d t}=B q N-\frac{q}{t_{c}},
\end{aligned}
$$

where $R$ is the pumping rate (atoms/second) into the upper laser level, $\tau^{-1}$ is the inversion relaxation rate, and $B$ is a constant proportional to the spontaneous transition rate such that $B q N$ is equal to the rate of induced transitions. The steady-state solutions of (4) and (5) for the case of constant photon losses $\left(1 / t_{c}=1 / t_{c o}\right)$ are

$$
\begin{gathered}
N_{0}=\frac{1}{B t_{c o}} \\
q_{0}=\frac{R B t_{c o}-1 / \tau}{B} \equiv \frac{1}{B \tau}(r-1) \\
r=R B t_{c o} \tau .
\end{gathered}
$$

Equation (6) has physical significance only for $r>1$. At threshold $r=1$ and for finite power output $(q>0), r>1$. The parameter $r$ is thus the factor by which the pumping rate exceeds its threshold value.

In considering the case of internal coupling modulation, we replace $t_{c}{ }^{-1}$ in (5) by

$$
\frac{1}{t_{c}(t)}=\frac{1}{t_{c o}}+\alpha(t)
$$

where $\alpha(t) \ll<t_{c o}{ }^{-1}$ represents the time-dependent part of the losses due to the modulation voltage as in (3). We write the solutions of (4) and (5) in this case as

$$
\begin{aligned}
N & =N_{0}+N_{1}(t), & & N_{1} \\
q & =q_{0}+N_{0}(t), & q_{\mathrm{I}} & \ll q_{0},
\end{aligned}
$$

where $N_{0}$ and $q_{0}$ are the steady-state solutions $(\alpha=0)$ as given by (6). Substituting (7) and (8) in the rate equations (4) and (5) and using (6) to eliminate the steady-state variables leads to

$$
\begin{aligned}
\frac{d N_{1}}{d t} & =-\frac{r}{\tau} N_{1}-\frac{q_{1}}{t_{c o}} \\
\frac{d q_{1}}{d t} & =\frac{1}{\tau}(r-1) N_{1}-\frac{(r-1)}{B \tau} \alpha(t) .
\end{aligned}
$$

In obtaining the last pair of equations we discarded second-order terms involving the factors $q_{1} N_{1}$ and $q_{1} \alpha$. From (9) and (10) we obtain

$\frac{d^{2} q_{1}}{d t^{2}}+\frac{r}{\tau} \frac{d q_{1}}{d t}+\frac{(r-1)}{\tau t_{c o}} q_{1}=-\frac{(r-1)}{B \tau} \frac{d \alpha}{d t}-\frac{r(r-1)}{B \tau^{2}} \alpha$.

The left-hand side of this equation is formally identical to that of a damped harmonic oscillator. The right-hand side represents the driving "force" due to the loss modulation $\alpha$. For the case of harmonic modulation we take $\alpha$ in the form

$$
\alpha(t)=\frac{\alpha_{1}}{2}\left(e^{i \omega t}+e^{-i \omega t}\right)
$$

and

$$
q_{1}=\frac{Q_{1}}{2}\left(e^{i \omega t}+e^{-i \omega t}\right),
$$

where, using (3)

$$
\alpha_{1}=\frac{2 c \Gamma_{0} \Gamma_{1}}{L}
$$

Substitution of (12) and (13) in (11) leads to

$$
Q_{1}=\frac{\frac{i \omega(r-1)}{B \tau}+\frac{r(r-1)}{B \tau^{2}}}{\left(\omega-\omega_{m}-i \beta\right)\left(\omega+\omega_{m}-i \beta\right)} \alpha_{1},
$$

where

$$
\begin{aligned}
\omega_{m} & \equiv \sqrt{\frac{1}{t_{c o} \tau}(r-1)-\left(\frac{r}{2 \tau}\right)^{2}} \\
\beta & \equiv \frac{r}{2 \tau} .
\end{aligned}
$$

From (15), it follows that at modulation frequencies $\omega$ $\approx \omega_{m}$, the laser-stored energy can oscillate strongly, while at $\omega-\omega_{m} \gg \beta, Q_{1}$ is small and $q(t) \rightarrow q_{0}$. This oscillation, in turn, will lead to a distortion of the power coupled out of the laser resonator. This problem is considered next.

The instantaneous fraction of the internal power coupled by the modulator-polarizer combination was given in (2) as

$$
T(t)=\sin ^{2} \Gamma(t) \approx \Gamma_{0}{ }^{2}+2 \Gamma_{0} \Gamma_{1} \cos \omega t, \quad \Gamma_{1}{ }^{2} \ll<\Gamma_{0}{ }^{2} .
$$

The number of photons per second coupled is thus

$$
\begin{aligned}
P(t)=\frac{c}{L} q(t) T(t)=\frac{c}{L}\left(q_{0}\right. & \left.+\frac{Q_{1}}{2} e^{i \omega t}+\frac{Q_{1}{ }^{*}}{2} e^{-i \omega t}\right) \\
& \cdot\left(\Gamma_{0}{ }^{2}+\Gamma_{0} \Gamma_{1} e^{i \omega t}+\Gamma_{0} \Gamma_{1} e^{-i \omega t}\right) .
\end{aligned}
$$

Singling out the terms with $\exp (i \omega t)$ time dependence gives

$$
P^{(\omega)}(t)=\frac{c}{L}\left(q_{0} \Gamma_{0} \Gamma_{1} e^{i \omega t}+\frac{Q_{1}}{2} \Gamma_{0}^{2} e^{i \omega t}+\text { c.c. }\right)
$$

for the component of the output power modulated at the 
signal frequency. The first term on the right side of (19) represents the useful undistorted output since according to (1) it is proportional to the modulation voltage amplitude $V_{1}=\Gamma_{1} V_{\pi} / \pi$. The second term, which is proportional to $Q_{1} \Gamma_{0}{ }^{2}$, represents a distortion of the output since, as shown in (15), $Q_{1}$ depends on the modulation frequency $\omega$. The distorted component is expected to be appreciable at modulation frequencies near $\omega_{m}$ where, according to (15), $Q_{1}$ goes through a maximum.

In order to obtain a better estimate of the expected distortion we use (6), (14), and (15) to write the last equation as

$$
\begin{aligned}
& P^{(\omega)}(t)=\left(\frac{c}{L}\right) \frac{\Gamma_{0} \Gamma_{1}(r-1)}{B \tau} \\
& {\left[1+\frac{\left(i \omega+\frac{r}{\tau} \Gamma_{0}^{2}\left(\frac{c}{L}\right)\right.}{2\left(\omega-\omega_{m}-i \beta\right)\left(\omega+\omega_{m}-i \beta\right)}\right] e^{i \omega t}+\text { c.c. }}
\end{aligned}
$$

The second term in the square brackets in (20) undergoes a rapid phase variation near $\omega=\omega_{m}$ so that a plot of $P^{(\omega)}(t)$ versus $\omega$ may be expected to display a structure near $\omega_{m}$, which is due to the interference of the amplitudes represerited by the two terms within the brackets.

\section{The Experiment and Experimental Results}

The experimental arrangement used for low-frequency coupling measurements is shown schematically in Fig. 1. The laser-discharge tube is of metal-ceramic construction with an active region $30 \mathrm{~cm}$ long by $5 \mathrm{~mm}$ in diameter. Power input is $33 \mathrm{~W}$ and heat is removed conductively to a water-cooled base and support structure. The tube is designed to operate in a hemiconfocal cavity $50 \mathrm{~cm}$ long with a TEM ${ }_{00}$ diffraction loss of about 2 percent. In this configuration, an output power of $3.6 \mathrm{~W}$ is obtained using an 85 -percent reflective output mirror. For this experiment, the laser is operated with fully reflective mirrors whose spacing can be varied between 40 and $90 \mathrm{~cm}$. The output power is coupled from the optical cavity via the GaAs Brewster window nearest the modulator. The modulator is a [110] oriented CdTe crystal $2 \mathrm{~cm}$ long by $5 \mathrm{~mm}^{2}$ with antireflection-coated faces that reduce the reflective insertion loss to less than 4 percent. The half-wave voltage (unclamped) for the modulator is $12 \mathrm{kV}$ at $10.6 \mu \mathrm{m}$. Fig. 2 shows the laser with the modulator installed in the optical cavity.

The modulator circuit is arranged to allow both ac and de signals to be applied simultaneously and independently, thus providing separate control of the parameters $\Gamma_{0}$ and $\Gamma_{1}$. The video driver amplifier is capable of $600 \mathrm{~V}$ peak to peak over a $4-\mathrm{MHz}$ bandwidth and the dc voltage is limited to $2 \mathrm{kV}$ to prevent dielectric breakdown. The corresponding (maximum) retardations are $\Gamma_{0}=0.52 \mathrm{rad}$ and $\Gamma_{1}=0.15 \mathrm{rad}$. A video sweep generator was used to drive

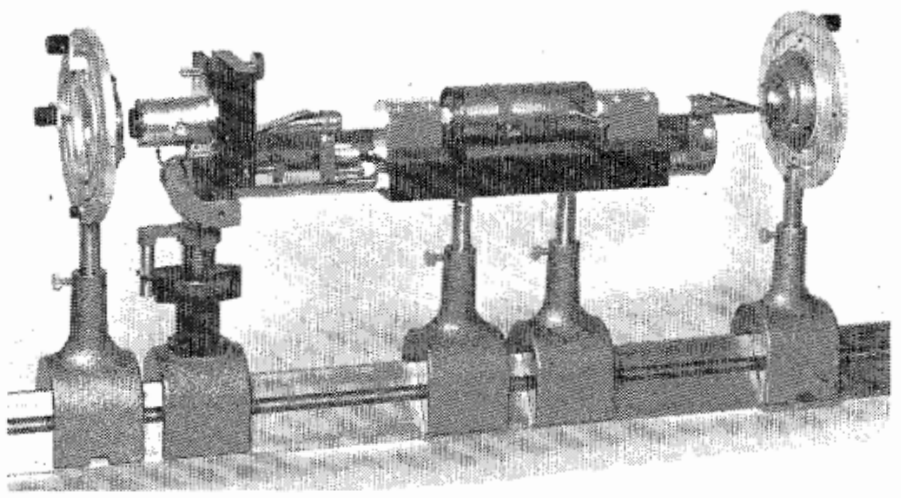

Fig. 2. $\mathrm{CO}_{2}$ laser with coupling modulator installed.

the amplifier and the horizontal input to an oscilloscope; the coupled output from the laser was detected, amplified, envelope detected, and displayed vertically.

The most important result of the theoretical analysis is (20) for the frequency dependence of the modulated power output. In our experiment, as discussed below, $\omega_{m}$ exceeds $r / \tau$ by nearly two orders of magnitude so that for frequencies $\omega>0.1 \omega_{m}$ we can approximate (20) by

$P^{(\omega)}=\left(\frac{c}{L}\right) \frac{\Gamma_{0} \Gamma_{1}(r-1)}{B \tau}\left[1-\frac{i \omega \Gamma_{0}{ }^{2}\left(\frac{c}{L}\right)}{2\left(i \beta+\omega_{m}-\omega\right)\left(\omega+\omega_{m}\right)}\right]$,

where $P^{(\omega)}$ is the complex amplitude of $P^{(\omega)}(t)$.

At $\omega=\omega_{m}$ the modulated component of the power output should have a minimum value of

$$
P^{\left(\omega_{m}\right)} \quad\left(\frac{c}{L}\right) \frac{\Gamma_{0} \Gamma_{1}(r-1)}{B \tau}\left[1-\frac{\omega \Gamma_{0}{ }^{2}\left(\frac{c}{L}\right)}{4 \beta \omega_{m}}\right] .
$$

The experimentally observed dip in the power output is shown in Fig. 3(a) and (b). The minimum of Fig. 3(a) occurs at a value of $f_{m} \equiv \omega_{m} / 2 \pi=2.1 \times 10^{5} \mathrm{~Hz}$. The theoretical value of $f_{m}$ was calculated using (16). The collision lifetime $\tau$ was obtained by using published [6] collision cross sections for $\mathrm{CO}_{2}$ molecules with $\mathrm{CO}_{2}, \mathrm{H}_{2}, \mathrm{He}$, and $\mathrm{N}_{2}$ molecules and the appropriate partial pressures $(8$, $0.2,15$, and 4 torr, respectively) of these constituents in our laser. The result is $\tau=7 \times 10^{-5} \mathrm{~s}$. The photon lifetime $t_{c o}$ appropriate to Fig. 3(a) is $t_{c o} \cong l / c(1-R) \approx 10^{-8} \mathrm{~s}$. The laser was pumped at twice its threshold value to $r=2$. Using these data in (16) gives

$$
\left(f_{m}\right)_{\text {theoretical }}=1.9 \times 10^{5} \mathrm{~Hz}
$$

to be compared with the observed value of $2.1 \times 10^{5} \mathrm{~Hz}$. As a further check, we compared the measured dependence of the dip frequency $f_{m}$ on the cavity length to the theoretical prediction of $f_{m} \propto l^{-1 / 2}$ that results from (16) when $t_{c o}<\tau$. This should result in a value of $f_{m}$ in Fig. 


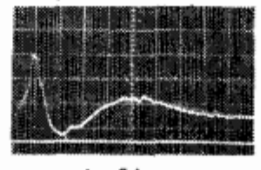

(a)

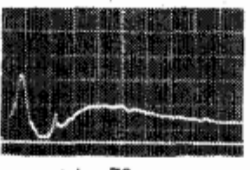

(b)
Fig. 3. Laser output power versus modulation frequency for two cavity lengths. Horizontal scale: $10 \mathrm{kHz} /$ div. Bias $=500$, signal $=600 \mathrm{~V}$ peak to peak.

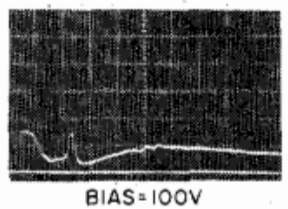

(a)

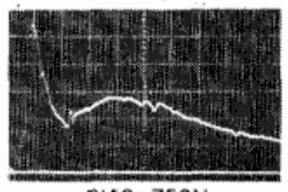

(c)

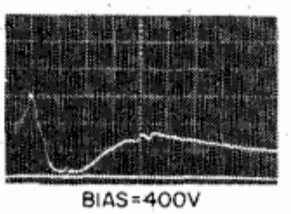

(b)

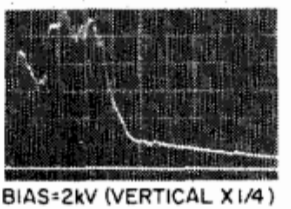

(d)
Fig. 4. Laser output power versus modulation frequency for four values of dc bias $\Gamma_{0}$. Horizontal scale: $100 \mathrm{kHz} /$ div. Signal $=600$ $\mathrm{V}$ peak to peak.

3(b) $(L=75 \mathrm{~cm})$, which is lower by a factor $\approx \sqrt{ } 1.4$ than the value of Fig. $3(\mathrm{a})(L=54 \mathrm{~cm})$. The observed change, from $2.1 \pm 0.1 \times 10^{5} \mathrm{~Hz}$ to $1.7 \pm 0.1 \times 10^{5} \mathrm{~Hz}$, is consistent with this prediction.

Another feature of the theory that agrees, at least qualitatively with the analysis, has to do with the dependence on the dip at $\omega=\omega_{m}$ on the dc retardation bias $\Gamma_{0}$. According to (22), an increase from zero of $\Gamma_{0}$ should lead to a decrease in the modulated component of the power output $\left.P^{\left(\omega_{m}\right)}\right)$ at the dip frequency. When $\Gamma_{0}{ }^{2}=$ $(4 \beta L / c)$ the output power is zero. Further increases in $\Gamma_{0}$ cause $P^{\left(\omega_{m}\right)}$ to increase.

This general behavior can be observed in the sequence of Fig. 4(a)-(d). The output $P^{\left(\omega_{m}\right)}$ becomes zero at a bias of $400 \mathrm{~V}$ and increases progressively with further increases in bias.

As a last check, we note in the sequence of Fig. 5(a)-(d) that the dip at $\omega=\omega_{m}$ is not affected by increasing the RF component of the retardation $\Gamma_{1}$. Away from $\omega_{m}$, but still

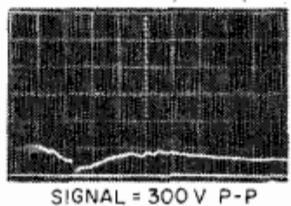

(a)

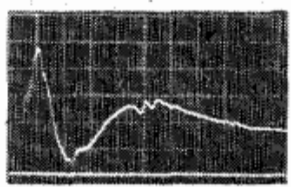

SIGNAL $=500 \mathrm{VP}-\mathrm{P}$

(c)

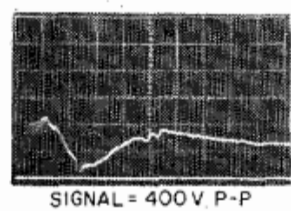

(b)

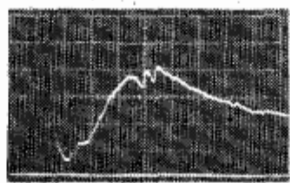

SIGNAL $=600 \mathrm{VP}-\mathrm{P}$

(d)
Fig. 5. Laser output power versus modulation frequency for different amplitudes of the modulation signal amplitude. Horizontal scale: 100 $\mathrm{kHz} /$ div. Bias $=500 \mathrm{~V}$.

at nearby frequencies, we notice a faster increase in the signal level than the proportional dependence on $\Gamma_{1}$ predicted by (22).

\section{Summary and Conclusions}

A region of severe distortion has been observed in the otherwise flat frequency response of intracavity laser coupling modulation. This anomalous behavior has been analyzed and explained in terms of a fundamental resonance in which the stored energy oscillates between the inverted atoms and the electromagnetic field.

The effect of this phenomenon is to limit the useful range of the coupling modulator to those frequencies well above those of the distortion region. In practice, it has been found that this useful range extends above $1 \mathrm{MHz}$.

\section{REFERENCES}

[1] K. Gurs and R. Muller, "Internal modulation of optical masers," Phys. Lett., vol. 5, p. 179, 1963.

[2] I. P. Kaminow, "Internal modulation of optical masers (bandwidth limitations)," Appl. Opt., vol. 4, p. 123, 1965.

[3] M. DiDomenico, "Small-signal analysis of internal (coupling-type) modulation of Lasers," J. Appl. Phys., vol. 35, pp. 2870-2876, 1964.

[4] F. R. Nash and P. W. Smith, "Broad-band optical coupling modulation," IEEE J. Quantum Electron., vol. QE-4, pp. 26-34, Jan. 1968.

[5] J. E. Kiefer, T. A. Nussmeier, and F. E. Goodwin, "Intracavity CdTe modulators for $\mathrm{CO}_{2}$ lasers," IEEE J. Quantum Electron., vol. QE-8, pp. $173-179,1972$.

[6] R. L. Taylor and S. Bitterman, "Survey of vibrational relaxation data for processes important in the $\mathrm{CO}_{2}-\mathrm{N}_{2}$ laser system," Rev. Modern Phys., vol. 41, p. 26, 1969. 